\title{
Correlation and Path Analysis Studies in Barley (Hordeum vulgare L.) Genotypes under Normal and Limited Moisture Conditions
}

\author{
Jaya Shrimali $^{1}$, A.S. Shekhawat ${ }^{1}$ and Sunita Kumari ${ }^{2 *}$ \\ ${ }^{1}$ Department of Plant Breeding and Genetics, College of Agriculture, Bikaner-334006, India \\ ${ }^{2}$ Department of Genetics and Plant Breeding, Institute of Agricultural Sciences, Banaras Hindu \\ University, Varanasi-22105, India \\ *Corresponding author
}

\section{A B S T R A C T}

Keywords

Barley,

Correlation and Path analysis, Randomize block design.

Article Info

Accepted: 19 June 2017 Available Online: 10 August 2017
This research was carried out the correlation among the yield and yield attributing characters and their path analysis for 30 genotypes of Barley in a Randomize Block Design (RBD) with three replications in two environments. The seed yield per plant was found to be positively and significantly associated with plant height, biological yield per plant, test weight, number of spikelets per spike and spike length in both the environments. Path coefficient analysis indicated that biological yield per plant and harvest index in both the environments, were the important characters for selection of high yielding genotype as this exerted high positive direct effect as well as showed high and positive correlation with seed yield.

\section{Introduction}

Barley (Hordeum vulgare L.) is the world's fourth most important cereal crop after wheat, maize and rice. Barley belongs to the family poaceae with chromosome number $2 \mathrm{n}=2 \mathrm{x}=14$ Barley is considered as most cosmopolitan of the crops, grown over the wide environmental range as well as it has been considered, as poor man's crop because of its low input requirement and better adaptability to harsh environments, like drought, salinity and alkalinity and marginal lands. Owing to its hardiness, in many countries around the world, it is often considered the only possible rainfed cereal crop under low input and stressful environments.
In any breeding programmed aiming at improving yield, it is essential to know, the degree of association between yield and other metric traits. Yield is complex character, which is contributed by many independent characters and improvement in yield depends upon improvement in its component characters. Correlation coefficient ensures the degree of association, genetic or non genetic relationship between two or more characters which forms the basis for selection. When change in one variable causes the change in another variable, the variables are said to be correlated. This association between the attributes is measured as "correlation 
coefficient". If the change is in same direction, the correlation is positive and if it is in opposite direction, the correlation is negative. The value is zero when two variables are not related. In plant breeding, study of correlation is essential because most of the traits such as yield are the end products of interaction of several genetic factors among themselves and their individual and combined interaction with environmental factors. Path analysis simply splits the correlation coefficient into the measures of direct and indirect effect of a set of independent variables on the dependent variables.

\section{Materials and Methods}

The germplasm of 30 genotypes was studied at Research Farm of College of Agriculture, Beechwal, Bikaner during rabi season 201213. Genotypes were obtained from Agriculture Research Station Durgapura, Jaipur, which were differing in growth and morphological characters. The experiment was laid out in Randomized Block Design with three replications. Each genotype was sown in double row with spaced apart at $23 \mathrm{x}$ $10 \mathrm{~cm}$ in a plot of $4 \times 3 \mathrm{~m}$ size. The genotypes were evaluated in two environments i.e. (i) normal moisture condition and (ii) limited moisture condition.

\section{Normal moisture condition}

All the standard agricultural practices were followed to raise the good and healthy crop in normal environment.

\section{Limited moisture condition}

first irrigation was given at the time of sowing and second life saving irrigations was given after 30 days of sowing and after 80 of sowing. After that no irrigation was given. The sample size consisted of five plants, selected randomly from each plot in both environments. The observations on different characters plant height, number of effective tillers per plant, spike length, number of spikelet's per spike, test weight, seed yield per plant, biological yield per plant, harvest index, Membrane stability index, Relative water content and chlorophyll content except days to $50 \%$ flowering, days to maturity were recorded on the basis of five selected plants and averaged to obtain the mean.

Genotypic and phenotypic correlation coefficients of yield with the contributing characters and among themselves were calculated by using the genotypic and phenotypic variances and co-variances as described by Singh et al., (1985) and Searle (1961). The direct and indirect effects were estimated through path coefficient analysis as suggested by Wright (1921) and elaborated by Dewey et al., (1959).

\section{Results and Discussion}

\section{Character association analysis}

The correlations between all possible combinations among the characters were calculated under normal and limited moisture condition at phenotypic and genotypic level and are presented in tables 1 and 2 respectively. Perusal of the table indicates that the magnitude of genotypic correlation coefficient was higher for most of the character pairs than their respective values of phenotypic correlation coefficient, which may be ascribed to the low effect of environment on the character expression.

Under normal condition seed yield per plant had significant positive correlation with plant height ( $\left.\mathrm{rg}=0.917^{* *}, \mathrm{rp}=0.798^{* *}\right)$, biological yield per plant $\left(\mathrm{rg}=0.865^{* *}, \mathrm{rp}=0.782^{* *}\right)$, test weight $\left(\mathrm{rg}=0.846^{* *}, \quad \mathrm{rp}=0.679 * *\right)$, number of spikelet per spike $(\mathrm{rg}=0.679 * *$, $\mathrm{rp}=0.574 * *)$, spike length ( $\mathrm{rg}=0.524 * *$, $\left.\mathrm{rp}=0.473^{* *}\right)$ at both phenotypic and genotypic level respectively. 
Table.1 Phenotypic and genotypic correlation coefficients between different characters in barley under normal moisture condition

\begin{tabular}{|c|c|c|c|c|c|c|c|c|c|c|c|c|c|c|}
\hline Characters & & $\begin{array}{c}\text { Days to } \\
50 \% \\
\text { flowering }\end{array}$ & $\begin{array}{l}\text { Days to } \\
\text { maturity }\end{array}$ & $\begin{array}{c}\text { Plant } \\
\text { height } \\
(\mathrm{cm})\end{array}$ & $\begin{array}{c}\text { No. of } \\
\text { effective } \\
\text { tillers } \\
\text { per } \\
\text { plant }\end{array}$ & $\begin{array}{c}\text { Spike } \\
\text { length } \\
(\mathrm{cm})\end{array}$ & $\begin{array}{c}\text { Number } \\
\text { of } \\
\text { spikelets } \\
\text { per } \\
\text { spike } \\
\end{array}$ & $\begin{array}{c}\text { Test } \\
\text { weight } \\
\text { (g) }\end{array}$ & $\begin{array}{l}\text { Biologic } \\
\text { al yield } \\
\text { per } \\
\text { plant }(\mathrm{g})\end{array}$ & $\begin{array}{c}\text { Harvest } \\
\text { index } \\
(\%)\end{array}$ & $\begin{array}{c}\text { Relative } \\
\text { water } \\
\text { content } \\
(\%)\end{array}$ & $\begin{array}{l}\text { Membra } \\
\text { ne } \\
\text { stability } \\
\text { index }\end{array}$ & $\begin{array}{c}\text { Chlorophy } \\
\text { ll content } \\
(\mathbf{m g} / \mathrm{g})\end{array}$ & $\begin{array}{c}\text { Seed } \\
\text { yield per } \\
\text { plant }(\mathrm{g})\end{array}$ \\
\hline Days to $50 \%$ & $r_{p}$ & 1.000 & $0.551 * *$ & 0.038 & -0.102 & -0.044 & 0.051 & 0.185 & 0.041 & 0.275 & 0.080 & -0.035 & 0.092 & 0.183 \\
\hline $\begin{array}{l}\text { flowering } \\
\text { Days to maturity }\end{array}$ & $\begin{array}{l}r_{g} \\
r_{p}\end{array}$ & 1.000 & $\begin{array}{c}0.543 * * \\
1.000\end{array}$ & $\begin{array}{c}0.095 \\
-0.046\end{array}$ & $\begin{array}{l}-0.112 \\
-0.075\end{array}$ & $\begin{array}{l}-0.031 \\
-0.168\end{array}$ & $\begin{array}{l}0.074 \\
0.084\end{array}$ & $\begin{array}{c}0.220 \\
-0.040\end{array}$ & $\begin{array}{r}0.059 \\
-0.038\end{array}$ & $\begin{array}{l}0.308 \\
0.258\end{array}$ & $\begin{array}{l}0.017 \\
0.002\end{array}$ & $\begin{array}{l}-0.053 \\
-0.048\end{array}$ & $\begin{array}{l}0.142 \\
0.108\end{array}$ & $\begin{array}{l}0.187 \\
0.107\end{array}$ \\
\hline Plant height $(\mathrm{cm})$ & $\begin{array}{l}r_{g} \\
r_{p}\end{array}$ & & 1.000 & $\begin{array}{r}-0.019 \\
1.000\end{array}$ & $\begin{array}{c}-0.080 \\
0.310\end{array}$ & $\begin{array}{l}-0.168 \\
0.419^{*}\end{array}$ & $\begin{array}{c}0.053 \\
0.698 * *\end{array}$ & $\begin{array}{c}-0.034 \\
0.708 * *\end{array}$ & $\begin{array}{c}-0.027 \\
0.806^{* *}\end{array}$ & $\begin{array}{r}0.303 \\
-0.045\end{array}$ & $\begin{array}{l}-0.001 \\
-0.211\end{array}$ & $\begin{array}{c}-0.076 \\
0.302\end{array}$ & $\begin{array}{l}0.126 \\
0.253\end{array}$ & $\begin{array}{c}0.109 \\
0.798 * *\end{array}$ \\
\hline $\begin{array}{l}\text { No. of effective tillers } \\
\text { per plant } \\
\text { Spike length }\end{array}$ & $\begin{array}{l}r_{g} \\
r_{p} \\
r_{g} \\
r_{p}\end{array}$ & & & 1.000 & $\begin{array}{l}0.337 \\
1.000 \\
1.000\end{array}$ & $\begin{array}{c}0.441^{*} \\
0.445^{*} \\
0.498^{* *} \\
1.000\end{array}$ & $\begin{array}{c}0.803 * * \\
-0.026 \\
-0.033 \\
0.279 * *\end{array}$ & $\begin{array}{c}0.916^{* *} \\
0.044 \\
0.076 \\
0.303\end{array}$ & $\begin{array}{c}0.896 * * \\
0.216 \\
0.241 \\
0.329\end{array}$ & $\begin{array}{l}-0.049 \\
0.043 \\
0.063 \\
0.164\end{array}$ & $\begin{array}{c}-0.233 \\
-0.128 \\
-0.142 \\
0.035\end{array}$ & $\begin{array}{c}0.357 \\
0.444 * \\
0.503 * * \\
0.270\end{array}$ & $\begin{array}{l}0.320 \\
0.205 \\
0.151 \\
0.272\end{array}$ & $\begin{array}{c}0.917 * * \\
0.286 \\
0.330 \\
0.473 * *\end{array}$ \\
\hline $\begin{array}{l}\text { Number of spikelets } \\
\text { per spike } \\
\text { Test weight }\end{array}$ & $\begin{array}{l}r_{g} \\
r_{p} \\
r_{g} \\
r_{p} \\
r_{g}\end{array}$ & & & & & 1.000 & $\begin{array}{c}0.339 * * \\
1.000 \\
1.000\end{array}$ & $\begin{array}{c}0.380 * \\
0.579 * * \\
0.743 * * \\
1.000 \\
1.000\end{array}$ & $\begin{array}{c}0.362 * \\
0.596 * * \\
0.686 * * \\
0.696 * * \\
0.797 * *\end{array}$ & $\begin{array}{c}0.207 \\
-0.069 \\
-0.090 \\
-0.039 \\
0.000\end{array}$ & $\begin{array}{l}0.016 \\
-0.155 \\
-0.126 \\
-0.088 \\
-0.120\end{array}$ & $\begin{array}{c}0.289 \\
0.141 \\
0.164 \\
0.327 \\
0.381 *\end{array}$ & $\begin{array}{c}0.403 * \\
0.235 \\
0.345 \\
0.099 \\
0.182\end{array}$ & $\begin{array}{l}0.524 * * \\
0.574 * * \\
0.679 * * \\
0.679 * * \\
0.846 * *\end{array}$ \\
\hline $\begin{array}{l}\text { Biological yield per } \\
\text { plant } \\
\text { Harvest index }(\%)\end{array}$ & $\begin{array}{l}r_{p} \\
r_{g} \\
r_{p}\end{array}$ & & & & & & & & $\begin{array}{l}1.000 \\
1.000\end{array}$ & $\begin{array}{r}-0.353 \\
-0.357 \\
1.000\end{array}$ & $\begin{array}{l}-0.100 \\
-0.125 \\
-0.090\end{array}$ & $\begin{array}{l}0.229 \\
0.286 \\
0.149\end{array}$ & $\begin{array}{l}0.091 \\
0.088 \\
0.224\end{array}$ & $\begin{array}{c}0.782 * * \\
0.865 * * \\
0.286\end{array}$ \\
\hline Relative water content & $\begin{array}{l}r_{g} \\
r_{p}\end{array}$ & & & & & & & & & 1.000 & $\begin{array}{c}-0.144 \\
1.000\end{array}$ & $\begin{array}{c}0.154 \\
-0.178\end{array}$ & $\begin{array}{c}0.426 * \\
0.086\end{array}$ & $\begin{array}{c}0.148 \\
-0.166\end{array}$ \\
\hline$(\%)$ & $r_{g}$ & & & & & & & & & & 1.000 & -0.189 & 0.121 & -0.214 \\
\hline $\begin{array}{l}\text { Membrane stability } \\
\text { index }\end{array}$ & $\begin{array}{l}r_{p} \\
r_{g}\end{array}$ & & & & & & & & & & & $\begin{array}{l}1.000 \\
1.000\end{array}$ & $\begin{array}{l}0.225 \\
0.338\end{array}$ & $\begin{array}{c}0.334 \\
0.386^{*}\end{array}$ \\
\hline $\begin{array}{l}\text { Chlorophyll content } \\
(\mathrm{mg} / \mathrm{g})\end{array}$ & $\begin{array}{l}r_{p} \\
r_{g}\end{array}$ & & & & & & & & & & & & $\begin{array}{l}1.000 \\
1.000\end{array}$ & $\begin{array}{l}0.248 \\
0.344\end{array}$ \\
\hline $\begin{array}{l}\text { Seed yield per plant } \\
(\mathrm{g})\end{array}$ & $\begin{array}{l}r_{p} \\
r_{g}\end{array}$ & & & & & & & & & & & & & $\begin{array}{l}1.000 \\
1.000 \\
\end{array}$ \\
\hline
\end{tabular}

$*$, ** Significant at $5 \%$ and $1 \%$ level of significance, respectively 
Table.2 Phenotypic and genotypic correlation coefficients between different characters in barley under limited moisture condition

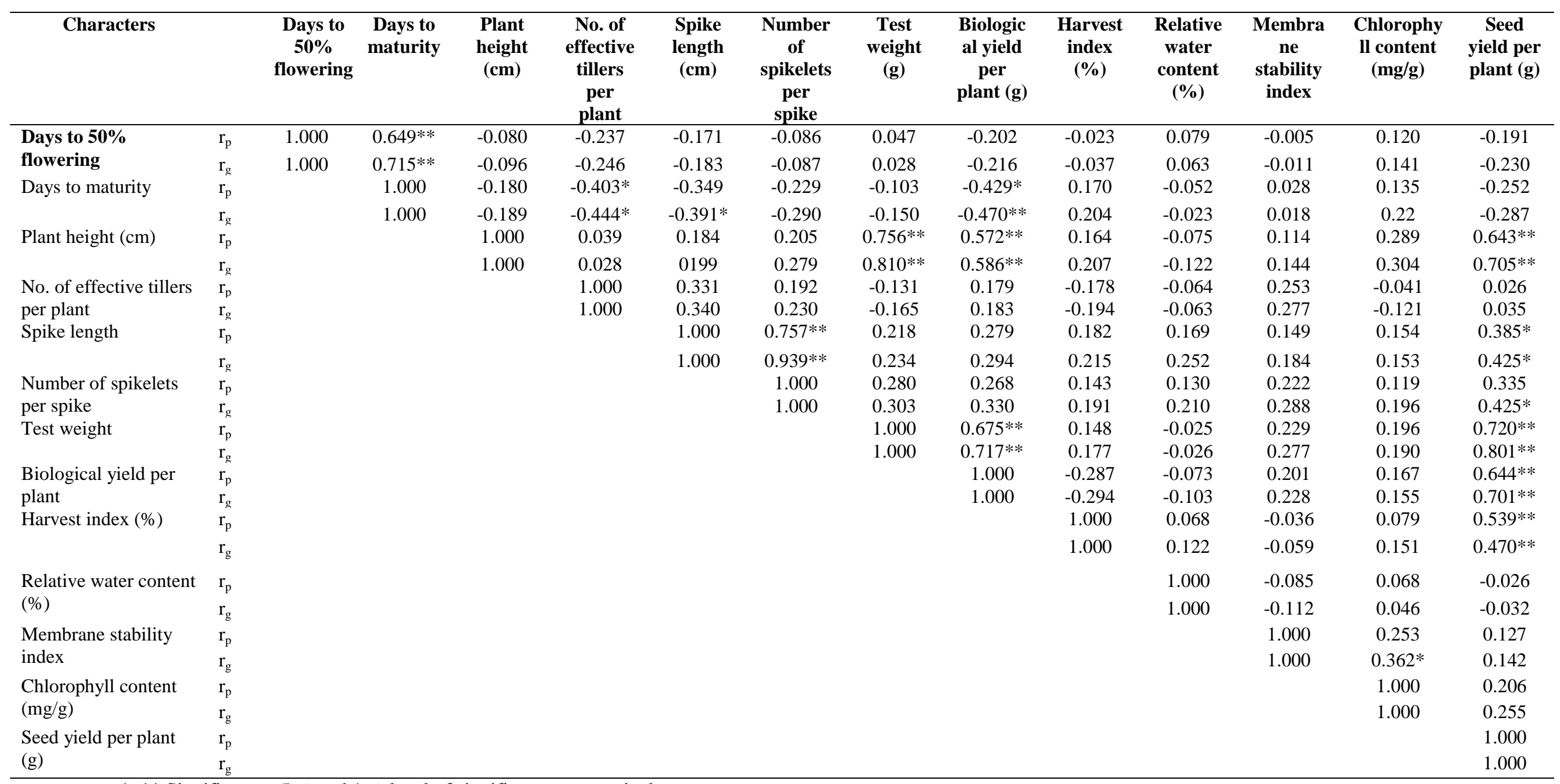

$*, * *$ Significant at $5 \%$ and $1 \%$ level of significance, respectively 
Table.3 Phenotypic (P) and genotypic (G) path coefficients of various characters on seed yield per plant under normal moisture condition

\begin{tabular}{|c|c|c|c|c|c|c|c|c|c|c|c|c|c|c|}
\hline Characters & & $\begin{array}{c}\text { Days to } \\
50 \% \\
\text { flowering }\end{array}$ & $\begin{array}{l}\text { Days to } \\
\text { maturity }\end{array}$ & $\begin{array}{c}\text { Plant } \\
\text { height } \\
(\mathrm{cm})\end{array}$ & $\begin{array}{c}\begin{array}{c}\text { No. of } \\
\text { effective } \\
\text { tillers }\end{array} \\
\text { per plant }\end{array}$ & $\begin{array}{c}\text { Spike } \\
\text { length } \\
(\mathrm{cm})\end{array}$ & $\begin{array}{c}\begin{array}{c}\text { Number } \\
\text { of } \\
\text { spikelets }\end{array} \\
\text { per spike }\end{array}$ & $\begin{array}{c}\text { Test } \\
\text { weight } \\
\text { (g) }\end{array}$ & $\begin{array}{l}\text { Biological } \\
\text { yield per } \\
\text { plant }(\mathrm{g})\end{array}$ & $\begin{array}{c}\text { Harvest } \\
\text { index } \\
(\%)\end{array}$ & $\begin{array}{c}\text { Relative } \\
\text { water } \\
\text { content } \\
(\%)\end{array}$ & $\begin{array}{l}\text { Membrane } \\
\text { stability } \\
\text { index }\end{array}$ & $\begin{array}{l}\text { Chlorophyll } \\
\text { content } \\
\text { (mg/g) }\end{array}$ & $\begin{array}{c}\text { Correlation } \\
\text { coefficient } \\
\text { with seed } \\
\text { yield per } \\
\text { plant }\end{array}$ \\
\hline \multirow{4}{*}{$\begin{array}{l}\text { Days to } 50 \% \\
\text { flowering } \\
\text { Days to maturity }\end{array}$} & $\mathbf{P}$ & -0.034 & 0.002 & 0.000 & -0.005 & -0.001 & 0.002 & 0.004 & 0.040 & 0.175 & 0.000 & 0.001 & 0.000 & 0.183 \\
\hline & $\mathbf{G}$ & 0.008 & 0.013 & 0.055 & 0.016 & -0.004 & -0.008 & -0.060 & 0.045 & 0.136 & 0.001 & -0.005 & -0.011 & 0.187 \\
\hline & $\mathbf{P}$ & -0.019 & 0.003 & 0.001 & -0.004 & -0.004 & 0.003 & -0.001 & -0.037 & 0.164 & 0.000 & 0.001 & 0.000 & 0.107 \\
\hline & $\mathbf{G}$ & 0.004 & 0.023 & -0.011 & 0.011 & -0.020 & -0.005 & 0.009 & -0.021 & 0.134 & 0.000 & -0.007 & -0.010 & 0.109 \\
\hline \multirow[t]{2}{*}{ Plant height $(\mathrm{cm})$} & $\mathbf{P}$ & -0.001 & 0.000 & -0.012 & 0.015 & 0.009 & 0.023 & 0.017 & 0.780 & -0.028 & 0.001 & -0.006 & 0.001 & $0.798 * *$ \\
\hline & $\mathbf{G}$ & 0.001 & 0.000 & 0.581 & -0.049 & 0.052 & -0.083 & -0.250 & 0.690 & -0.022 & -0.009 & 0.030 & -0.024 & $0.917 * *$ \\
\hline \multirow{4}{*}{$\begin{array}{l}\text { No. of effective } \\
\text { tillers per plant } \\
\text { Spike length }\end{array}$} & $\mathbf{P}$ & 0.003 & 0.000 & -0.004 & 0.048 & 0.009 & -0.001 & 0.001 & 0.209 & 0.028 & 0.001 & -0.009 & 0.001 & 0.286 \\
\hline & $\mathbf{G}$ & -0.001 & -0.002 & 0.196 & -0.144 & 0.059 & 0.003 & -0.021 & 0.186 & 0.028 & -0.006 & 0.043 & -0.012 & 0.330 \\
\hline & $\mathbf{P}$ & 0.001 & 0.000 & -0.005 & 0.021 & 0.021 & 0.009 & 0.007 & 0.319 & 0.104 & 0.000 & -0.006 & 0.001 & $0.473 * *$ \\
\hline & $\mathbf{G}$ & 0.000 & -0.004 & 0.256 & -0.072 & 0.119 & -0.035 & -0.104 & 0.279 & 0.091 & 0.001 & 0.025 & -0.031 & $0.524 * *$ \\
\hline \multirow{2}{*}{$\begin{array}{l}\text { Number of } \\
\text { spikelets per spike }\end{array}$} & $\mathbf{P}$ & -0.002 & 0.000 & -0.008 & -0.001 & 0.006 & 0.033 & 0.014 & 0.577 & -0.044 & 0.001 & -0.003 & 0.001 & $0.574 * *$ \\
\hline & $\mathbf{G}$ & 0.001 & 0.001 & 0.467 & 0.005 & 0.040 & -0.104 & -0.203 & 0.529 & -0.040 & -0.005 & 0.014 & -0.026 & $0.679 * *$ \\
\hline \multirow{2}{*}{$\begin{array}{l}\text { Biological yield } \\
\text { per plant }\end{array}$} & $\mathbf{P}$ & -0.001 & 0.000 & -0.009 & 0.010 & 0.007 & 0.019 & 0.017 & 0.968 & -0.224 & 0.001 & -0.005 & 0.000 & $0.782 * *$ \\
\hline & $\mathbf{G}$ & 0.000 & -0.001 & 0.521 & -0.035 & 0.043 & -0.071 & -0.218 & 0.771 & -0.158 & -0.005 & 0.024 & -0.007 & $0.865 * *$ \\
\hline \multirow[t]{2}{*}{ Harvest index (\%) } & $\mathbf{P}$ & -0.009 & 0.001 & 0.001 & 0.002 & 0.003 & -0.002 & -0.001 & -0.342 & 0.636 & 0.001 & -0.003 & 0.001 & 0.286 \\
\hline & $\mathbf{G}$ & 0.002 & 0.007 & -0.029 & -0.009 & 0.024 & 0.009 & 0.000 & -0.275 & 0.442 & -0.006 & 0.013 & -0.032 & 0.148 \\
\hline \multirow{2}{*}{$\begin{array}{l}\text { Relative water } \\
\text { content (\%) }\end{array}$} & $\mathbf{P}$ & 0.000 & 0.000 & 0.002 & -0.006 & 0.001 & -0.005 & -0.002 & -0.097 & -0.057 & -0.006 & 0.004 & 0.000 & -0.166 \\
\hline & $\mathbf{G}$ & 0.000 & 0.000 & -0.135 & 0.020 & 0.002 & 0.013 & 0.033 & -0.097 & -0.064 & 0.039 & -0.016 & -0.009 & -0.214 \\
\hline \multirow{2}{*}{$\begin{array}{l}\text { Membrane } \\
\text { stability index }\end{array}$} & $\mathbf{P}$ & 0.001 & 0.000 & -0.004 & 0.021 & 0.006 & 0.005 & 0.008 & 0.222 & 0.094 & 0.001 & -0.021 & 0.001 & 0.334 \\
\hline & $\mathbf{G}$ & 0.000 & -0.002 & 0.207 & -0.073 & 0.034 & -0.017 & -0.104 & 0.221 & 0.068 & -0.007 & 0.085 & -0.026 & $0.386^{*}$ \\
\hline \multirow{2}{*}{$\begin{array}{l}\text { Chlorophyll } \\
\text { content (mg/g) }\end{array}$} & $\mathbf{P}$ & -0.003 & 0.000 & -0.003 & 0.010 & 0.006 & 0.008 & 0.002 & 0.088 & 0.142 & 0.000 & -0.005 & 0.003 & 0.248 \\
\hline & G & 0.001 & 0.003 & 0.186 & -0.022 & 0.048 & -0.036 & -0.050 & 0.068 & 0.189 & 0.005 & 0.029 & -0.076 & 0.344 \\
\hline
\end{tabular}

Residual effect : Phenotypic $=0.0227$ and Genotypic $=0.0195$ 
Table.4 Phenotypic (P) and genotypic (G) path coefficients of various characters on seed yield per plant under limited moisture condition

\begin{tabular}{|c|c|c|c|c|c|c|c|c|c|c|c|c|c|c|}
\hline Characters & & $\begin{array}{c}\text { Days to } \\
50 \% \\
\text { flowering }\end{array}$ & $\begin{array}{l}\text { Days to } \\
\text { maturity }\end{array}$ & $\begin{array}{c}\text { Plant } \\
\text { height } \\
(\mathrm{cm})\end{array}$ & $\begin{array}{c}\text { No. of } \\
\text { effective } \\
\text { tillers } \\
\text { per plant }\end{array}$ & $\begin{array}{c}\text { Spike } \\
\text { length } \\
(\mathrm{cm})\end{array}$ & $\begin{array}{c}\begin{array}{c}\text { Number } \\
\text { of } \\
\text { spikelets } \\
\text { per spike }\end{array} \\
\end{array}$ & $\begin{array}{c}\text { Test } \\
\text { weight } \\
\text { (g) }\end{array}$ & $\begin{array}{l}\text { Biological } \\
\text { yield per } \\
\text { plant }(\mathrm{g})\end{array}$ & $\begin{array}{c}\text { Harvest } \\
\text { index } \\
(\%)\end{array}$ & $\begin{array}{c}\text { Relative } \\
\text { water } \\
\text { content } \\
(\%)\end{array}$ & $\begin{array}{l}\text { Membr } \\
\text { ane } \\
\text { stability } \\
\text { index }\end{array}$ & $\begin{array}{l}\text { Chloro } \\
\text { phyll } \\
\text { content } \\
(\mathrm{mg} / \mathrm{g})\end{array}$ & $\begin{array}{c}\text { Correlation } \\
\text { coefficient } \\
\text { with seed } \\
\text { yield per } \\
\text { plant }\end{array}$ \\
\hline \multirow{4}{*}{$\begin{array}{l}\text { Days to } 50 \% \\
\text { flowering } \\
\text { Days to maturity }\end{array}$} & $\mathbf{P}$ & 0.013 & -0.019 & 0.000 & -0.006 & -0.002 & 0.002 & 0.003 & -0.165 & -0.018 & -0.002 & 0.000 & 0.001 & -0.191 \\
\hline & $\mathbf{G}$ & -0.070 & 0.023 & 0.012 & -0.029 & 0.004 & -0.002 & 0.008 & -0.162 & -0.025 & -0.003 & 0.001 & 0.012 & -0.230 \\
\hline & $\mathbf{P}$ & 0.008 & -0.029 & 0.001 & -0.010 & -0.003 & 0.005 & -0.007 & -0.350 & 0.132 & 0.001 & -0.001 & 0.001 & -0.252 \\
\hline & $\mathbf{G}$ & -0.050 & $\mathbf{0 . 0 3 3}$ & 0.024 & -0.053 & 0.009 & -0.007 & -0.043 & -0.353 & 0.136 & 0.001 & -0.002 & 0.019 & -0.287 \\
\hline Plant height $(\mathrm{cm})$ & $\mathbf{G}$ & 0.007 & -0.006 & -0.127 & 0.003 & -0.004 & 0.006 & 0.234 & 0.440 & 0.138 & 0.006 & -0.018 & 0.026 & $0.705 * *$ \\
\hline \multirow{4}{*}{$\begin{array}{l}\text { No. of effective } \\
\text { tillers per plant } \\
\text { Spike length }\end{array}$} & $\mathbf{P}$ & -0.003 & 0.012 & 0.000 & 0.025 & 0.003 & -0004 & -0.008 & 0.146 & -0.138 & 0.001 & -0.007 & 0.000 & 0.026 \\
\hline & $\mathbf{G}$ & 0.017 & -0.014 & -0.004 & 0.120 & -0.007 & 0.005 & -0.048 & 0.137 & -0.129 & 0.003 & -0.035 & -0.010 & 0.035 \\
\hline & $\mathbf{P}$ & -0.002 & 0.010 & -0.001 & 0.008 & 0.009 & -0.016 & 0.014 & 0.228 & 0.141 & -0.004 & -0.004 & 0.002 & $0.385^{*}$ \\
\hline & $\mathbf{G}$ & 0.013 & -0.013 & -0.025 & 0.041 & -0.022 & 0.022 & 0.068 & 0.221 & 0.143 & -0.012 & -0.023 & 0.013 & $0.425 *$ \\
\hline \multirow{3}{*}{$\begin{array}{l}\text { Number of } \\
\text { spikelets per spike } \\
\text { Test weight }\end{array}$} & $\mathbf{P}$ & -0.001 & 0.007 & -0.001 & 0.005 & 0.007 & -0.022 & 0.018 & 0.219 & 0.110 & -0.003 & -0.006 & 0.001 & 0.335 \\
\hline & $\mathbf{G}$ & 0.006 & -0.009 & -0.035 & 0.028 & -0.021 & 0.023 & 0.088 & 0.248 & 0.127 & -0.010 & -0.036 & 0.017 & $0.425^{*}$ \\
\hline & $\mathbf{G}$ & -0.002 & -0.005 & -0.103 & -0.020 & -0.005 & 0.007 & 0.289 & 0.539 & 0.118 & 0.001 & -0.035 & 0.016 & $0.801 * *$ \\
\hline \multirow{2}{*}{$\begin{array}{l}\text { Biological yield } \\
\text { per plant }\end{array}$} & $\mathbf{P}$ & -0.003 & 0.013 & -0.002 & 0.004 & 0.003 & -0.006 & 0.044 & 0.816 & -0.222 & 0.002 & -0.006 & 0.002 & $0.644 * *$ \\
\hline & $\mathbf{G}$ & 0.015 & -0.015 & -0.074 & 0.022 & -0.006 & 0.008 & 0.207 & 0.751 & -0.195 & 0.005 & -0.029 & 0.013 & $0.701 * *$ \\
\hline \multirow[t]{2}{*}{ Harvest index (\%) } & $\mathbf{P}$ & 0.000 & -0.005 & -0.001 & -0.004 & 0.002 & -0.003 & 0.010 & -0.234 & 0.774 & -0.001 & 0.001 & 0.001 & $0.539 * *$ \\
\hline & $\mathbf{G}$ & 0.003 & 0.007 & -0.026 & -0.023 & -0.005 & 0.004 & 0.051 & -0.220 & 0.665 & -0.006 & 0.007 & 0.013 & $0.470 * *$ \\
\hline \multirow{2}{*}{$\begin{array}{l}\text { Relative water } \\
\text { content }(\%)\end{array}$} & $\mathbf{P}$ & 0.001 & 0.002 & 0.000 & -0.002 & 0.002 & -0.003 & -0.002 & -0.059 & 0.052 & -0.021 & 0.002 & 0.001 & -0.026 \\
\hline & $\mathbf{G}$ & -0.004 & -0.001 & 0.016 & -0.008 & -0.006 & 0.005 & -0.008 & -0.077 & 0.081 & -0.048 & 0.014 & 0.004 & -0.032 \\
\hline \multirow{2}{*}{$\begin{array}{l}\text { Membrane } \\
\text { stability index }\end{array}$} & $\mathbf{P}$ & 0.000 & -0.001 & 0.000 & 0.006 & 0.001 & -0.005 & 0.015 & 0.164 & -0.028 & 0.002 & -0.029 & 0.003 & 0.127 \\
\hline & G & 0.001 & 0.001 & -0.018 & 0.033 & -0.004 & 0.007 & 0.080 & 0.171 & -0.039 & 0.005 & -0.125 & 0.031 & 0.142 \\
\hline \multirow{2}{*}{$\begin{array}{l}\text { Chlorophyll } \\
\text { content (mg/g) }\end{array}$} & $\mathbf{P}$ & 0.002 & -0.004 & -0.001 & -0.001 & 0.001 & -0.003 & 0.013 & 0.136 & 0.061 & -0.001 & -0.007 & 0.011 & 0.206 \\
\hline & $\mathbf{G}$ & -0.010 & 0.007 & -0.039 & -0.014 & -0.003 & 0.005 & 0.055 & 0.116 & 0.100 & -0.002 & -0.045 & 0.086 & 0.255 \\
\hline
\end{tabular}

Residual effect: Phenotypic $=0.0119$ and Genotypic $=0.0013$ 
Under limited moisture condition seed yield per plant had positive and significant association with test weight $\left(\mathrm{rg}=0.801 * *, \mathrm{rp}=0.720^{* *}\right)$, plant height $\left(\mathrm{rg}=0.705^{* *}, \mathrm{rp}=0.643^{* *}\right)$, biological yield per plant $(\mathrm{rg}=0.701 * *, \mathrm{rp}=0.644 * *)$, harvest index $(\mathrm{rg}=0.470 * *, \mathrm{rp}=0.539 * *)$, spike length $\left(\mathrm{rg}=0.425^{*}, \mathrm{rp}=0.385^{*}\right)$ at both genotypic and phenotypic level, respectively. While, number of spikelet per spike $\left(\mathrm{rg}=0.425^{*}\right)$ had significant and positive association with seed yield per plant at genotypic level.

\section{Path coefficient analysis}

The direct and indirect effects of various characters along with their phenotypic correlation coefficients with seed yield per plant are presented in tables 3 and 4 for each of the two environmental conditions. Path coefficient analysis over two environmental conditions indicated the variable contribution of different characters towards seed yield with change of environmental conditions. Change in the diameter and magnitude of direct and indirect effects were noted between environments.

In normal condition the most of the traits which exhibited significant and positive correlation with seed yield per plant had high positive direct effects on seed yield per plant indicating that the major portion of correlation is ascribed to direct effects. Among the traits, biological yield per plant, plant height, harvest index, spike length and membrane stability index. Test weight exhibited high negative direct effect on seed yield per plant at genotypic level. The above findings are in conformity with (Najeeb et al., 2004; Kundalia et al., 2006; Mittal et al., 2009) in barley.

Similar to normal condition, the highest direct effect was exhibited by biological yield per plant, harvest index, test weight and number of effective tillers per plant in limited moisture condition. Such results were earlier reported by
(Vaezi et al., 2010; Ali et al., 2012; Naghaii et al., 2012).

\section{References}

Ali, I.H. and Shakor, E.F. 2012. Heritability, variability, genetic correlation and path analysis for quantitative traits in durum and bread wheat under dry farming conditions. Mesoptamia J. Agri., 40(4): 27-39.

Dewey, J.R. and Lu, K.H. 1959. A correlation and path coefficient analysis of components of crested wheat-grass seed production. Agron. J., 51: 515-518

Kundalia, S.K., Singh, P.P. and Solomon, S. 2006. Interrelationship of yields and associated characters in hull-less barley (Hordeum vulgare L.). Int. J. Plant Sci. Res., 33(1-4).

Mittal, V.P., Brar, K.S. and Singh, P. 2009. Interrelationships and path coefficient analysis for yield and component characters in barley (Hordeum vulgare L.). J. Agri. Sci., 5(1): 151-153.

Naghaii, Vali and Asgharipour, M.R. 2012. Difference in drought stress responses of 20 barley genotypes with contrasting drought tolerance during grain filling. Adv. Environ. Biol., 5(9): 3042-3049.

Najeeb, S. and Wani, S.A. 2004. Correlation and path analysis studies in barley (Hordeum vulgare L.). Nat. J. Plant-Improvement, 6(2): 124-125.

Searle, S.R. 1961. Biometrics, 17: 474-480.

Singh, R.K. and Choudhary, B.D. 1985. Biometrical Methods in Quantitative Genetic Analysis, Kalyani Publishers (Rev. Ed., 1985), Ludhiana, pp: 39-68.

Vaezi, B., Bavei, V. and Shiran, B. 2010. Screening of barley genotypes for drought tolerance by agro-physiological traits in field condition. African J. Agri. Res., 5(9): 881-892.

Wright, S. 1921. Correlation and causation. $J$. Agric. Res., (20): 557-587.

\section{How to cite this article:}

Jaya Shrimali, A.S. Shekhawat and Sunita Kumari. 2017. Correlation and Path Analysis Studies in Barley (Hordeum vulgare L.) Genotypes under Normal and Limited Moisture Conditions. Int.J.Curr.Microbiol.App.Sci. 6(8): 1850-1856.

doi: https://doi.org/10.20546/ijcmas.2017.608.218 